\title{
EL TERAPEUTA OCUPACIONAL Y LA REHABILITACIÓN EN SALUD MENTAL
}

" Habría que pensar más en términos de salud que en términos de enfermedad "

María Eugenia Caicedo Concha Terapéuta Ocupacional Mg. Psicología Comunitaria Decana Facultad de Terapia Ocupacional, U. Rosario

\section{Referentes Generales}

La rehabilitación en sentido amplio comprende tanto enfrentar las consecuencias de un trastorno como la reflexión alrededor del problema, lo que obviamente conduce no solo la busqueda de estrategias novedosas sino a encontar desde la perspectiva la identificación de los factores específicos necesarios para programas de promociòn, prevención y manejo comunitario como única forma de ampliar coberturas, racionalizar recursos y hacer a las comunidades responsables de su propia vida.

En lo referente a Terapia Ocupacional, la ocupacion del hombre se apoya sobre un tripode conformado por las habilidades y destrezas físicas, los factores psicosociales y las condiciones comunitarias. El terapeuta ocupacional ha sido el profesional que tradicionalmente se ha responsabilizado de la rehabilitación del enfermo mental y sus conocimientos y manejo sobre las condiciones ocupacionales lo convierten en uno de los mejor dotados a la hora de planificar programas que incluyan el aspecto psicosocial.

Pocos temas suscitan tanta controversia como la busqueda de sistemas de atención en salud mental, que permitan el cumplimiento del objetivo rehabilitador de facilitarle a la persona encontrar la manera de mejorar su propia vida.

Es evidente que en el discurso cada vez tiene más fuerza la idea de superar antiguas practicas de atención; es claro que cada vez se conocen más los derechos básicos de la persona con enfermedad mental según los cuales no habrá discriminación por enfermedad mental y la persona conserva sus derechos mientras se demuestre que no puede ocuparse de sus asuntos; nadie discute a nivel teórico que la Salud Mental es calidad de vida; no se requiere de mucha argumentación para demostrar que la insatisfacción de necesidades vitales mínimas acarrea muchas veces complicaciones mentales.

Los avances cientifico técnicos en las áreas de la biología,la psicolo- 
gia y la psiquiatría social son considerables, así como la comprensión del significado de la cultura y de la inequidad en Salud Mental, pero deberá avanzarse más en la corrección de las desigualdades arbitrarias y la satisfacción real de las necesidades de los usuarios, en la atención a los factores de riesgo y en la consideración de los factores psicosociales en cualquier programa de prevención y de atención en rehabilitación.

Aquí estamos hablando entonces de tres escenarios diferentes en Salud Mental que obviamente suponen estrategias distintas:

- Control de los trastornos psiquiátricos

- Promoción de la Salud Mental y control de los factores de riesgo en las poblaciones relacionadas con los procesos comunitarios.

- Aspectos de Salud Mental inherentes a todos los procesos de rehabilitación.

\subsection{Control de los Trastornos Psiquiátricos}

Actualmente encontramos que coexisten programas tradicionales en los cuales el hospital psiquiátrico es el centro y Terapia Ocupacional el nucleo del funcionamiento, apoyados en la concepción de que la ocupación promueve el contacto con la realidad y con los demás; pasando por alternativas de hospitalización y apoyos intermedios; programas basados en la segmentación de la población (por la edad o por otras características); hasta propuestas que apuntan al mejoramiento de las condiciones de vida de todo el grupo afectado respetando su cultura.

La consolidación progresiva de las unidades de Salud Mental en hospitales generales, los hospitales de día, las redes de servicios y los programas de reubicación laboral, los programas comunitarios, - sin contar con nuevas entidades clínicas como el aumento progresivo del estrés, la farmacodepedencia, enfermedades progresivas y degenerativas y el SIDA que probablemente requieran alternativas novedosas en su tratamiento - se convierten en un desafío para el rehabilitador que obviamente deberá adaptar sus prácticas.

Habría que revisar la actualización y productividad de algunos servicios particularmente el hospital psiquiátrico tradicional como eje de la atención, la diversificación en las alternativas de intervención y la articulación de los servicios ásí como la operatividad de los equipos profesionales actuales. Comienzan a aparecer ahora profesionales muy importantes en esta nueva perspectiva como los comunicadores, los nutricionistas, los planificadores y otros. 
En la búsqueda de nuevas alternativas de control a los problemas psiquiátricos, en lo que se refiere a la reorganización de los servicios para estimular su orientación hacia lo comunitario, descentralizado, participativo, integral y preventivo según lo plantea la declaración de Caracas (1990), todavia se cuestionan los logros de los procesos de desinstitucionalización y de atención comunitaria y periódicamente reaparece la tendencia hacia las antiguas prácticas de los hospitales psiquiátricos tradicionales, que facilitan un mayor control de los casos asignados.

En concordancia con el programa propuesto por la Organización Panamericana de la Salud los ejes para estos programas deberían fundamentarse en:

- Apoyo de las acciones de vigilancia para el cumplimiento de los derechos del enfermo mental.

- Fortalecimiento de una red de atención que incluya el sector salud y otros campos comprometidos (jurídico, económico, educación, comunicaciones, bienestar social).

- Acciones orientadas a promover alternativas de atención según la problemática donde el problema a enfrentar definiria las características del programa.

- Respaldo al desarrollo de investigaciones operativas.

- Acciones tendientes a adecuar a las nuevas tendencias, la formación de pre y postgrado, de los profesionales comprometidos.

\subsection{Promoción y Prevención}

La Salud Mental como tal es constitutiva de los sistemas de salud y en este sentido forma parte de todos los programas de promoción, prevención, tratamiento y rehabilitâción; no existe ninguna situación vital en el que el componente psicológico no tenga una amplia injerencia y por tanto un sentido global, cualquier programa de salud debería contemplar acciones para preservar o mejorar el quilibrio mental de las personas propendiendo asi por su calidad de vida; la salud y la enfermedad son de naturaleza integral y en su conservación el comportamiento juega un papel primordial.

La ley 100 de Seguridad Social reconoce en la promoción y la prevención la urgencia de identificar determinados aspectos del comportamiento (habitos de vida) definitivos en la salud y de las condiciones sociales 
y culturales determinantes en el binestar general de las poblaciones; se supone que en un mediano plazo la aplicación de estas medidas debe reflejarse en una disminución de las camas psiquiátricas requeridas y que estas serán destinadas progresivamente a los casos de mayor gravedad y a las crisis.

En la identificación y el manejo de estilos de vida saludables el terapeuta ocupacional tiene una formación privilegiada; estos hábitos están constituidos por lo que la gente hace en su vida diaria y por el significado que el atribuye a esas ocupaciones.

Se trata de que las comunidades sean capaces de favorecer o modificar su forma de vida para hacerse responsables de su salud y el terapeuta ocupacional resulta muy valioso siempre que sea capaz de analizar e intervenir en las ocupaciones rutinarias, considerando su marco cultural como explicativo del sentido y de las prácticas frente a la salud, la enfermedad y la muerte.

\subsection{Rehabilitación}

La rehabilitación en el sentido estricto de enfrentar la deficiencia y sus consecuencias a nivel del desempeño y de las relaciones con los demás (discapacidad y minusvalía) no puede ser entendida sin sus implicaciones psicosociales. Sin embargo en la rehabilitación el componente psicosocial es frecuentemente soslayado y además del estereotipado "apoyo psicológico" y de las medidas específicas adoptadas con poblaciones particulares no hemos avanzado mucho.

Sorprende encontrar todavía la dicotomía "lo físico y lo psicológico"; el desconocimiento de los aspectos psicológicos en una afección somática y desde la otra perspectiva que los terapeutas de las personas con discapacidad mental sean los únicos que todavia no diferencien entre la recuperación funcional, la reubicación profesional y la readaptación social.

En este sentido, el terapeuta ocupacional que interviene en el equilibrio de las rutinas ocupacionales lo está haciendo no solo a nivel de habilidades y destrezas sino también en la valoración de estas mismas rutinas y por supuesto de lo que estas adquisiciones significan para el grupo afectado.

\section{EL EQUIPO HUMANO Y SU FORMACION}

Un equipo de Salud Mental no es simplemente una yuxtaposición de profesionales que han desarrollado distintas habilidades, sino un conjunto integrado, con un nivel cualitativo y funcional superior que per- 
mite resolver efectivamente las situaciones que se van presentando, en las cuales el marco organizativo y de formas de trabajo es esencial para favorecer el proceso; en ellos el instrumento de trabajo son las mismas personas. Este equipo por tanto está funcionalmente integrado con el usuario del servicio y con la comunidad que lo requiere hasta el punto en que todos (personas y contexto) son el equipo.

La formación continuada de estos grupos se basa en un "saber hacer" y en la promoción de los conocimientos individuales para impulsar la transformación y la organización de nuevos servicios desplazando las actitudes rutinarias, con el sentimientos de pertenencia a un proyecto que amplie el horizonte profesional y cultural. Siendo asi los Terapeutas Ocupacionales como integrantes básicos del equipo deberían revisar desde los perfiles profesionales actuales, incluyendo las prácticas en que se forman; hasta sus rutinas fundamentales y los procesos investigativos que vienen adelantando.

Para responder a un programa de Salud Mental como el que propone la Organización Panamericana de la Salud vinculado con:

-La promoción de la Salud Mental y la prevención de los trastornos psiquiátricos

-El control de los trastornos psiquiátricos y neurológicos.

-Los factores psicosociales en la salud y en el desarrollo

Podemos utilizar el modelo de Goldberd y Huxley (1980) que propone una descentración de la atención del Hospital Psiquiátrico, hacia una

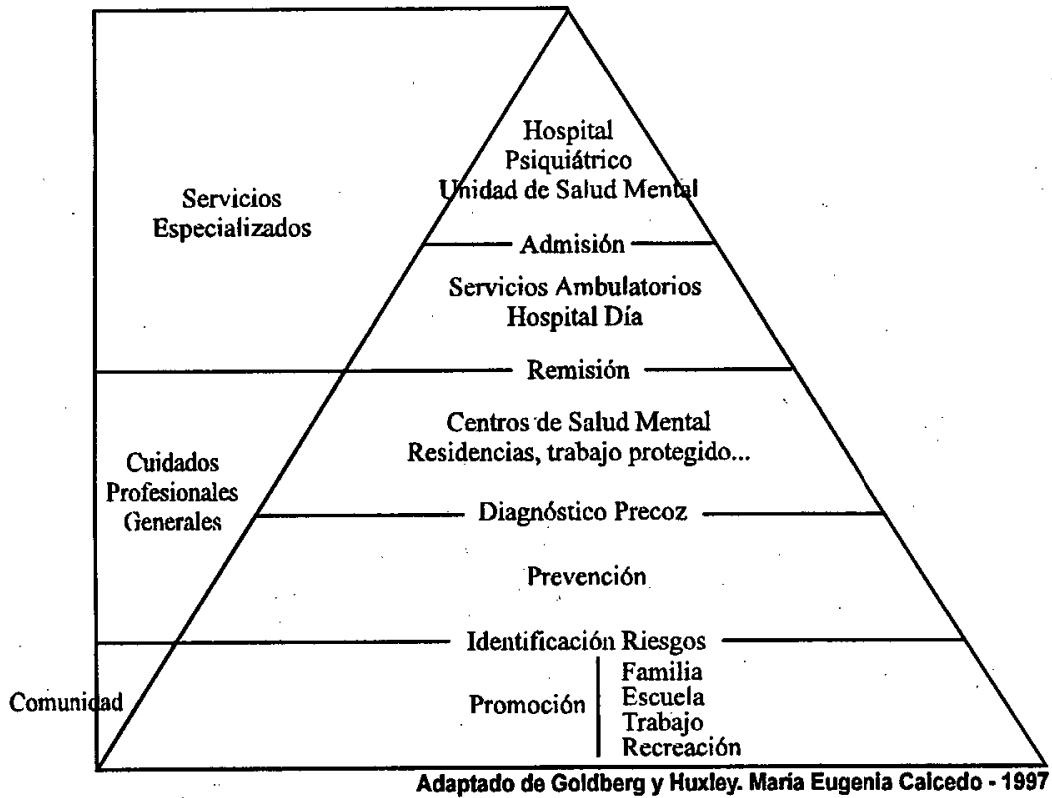


atención a la población desde programas de promoción y con filtros sucesivos para garantizar la utilización del servicio requerido y por ende la racionalización del recurso, para lo cual debería prepararse el Terapeuta Ocupacional en formación.

Siendo así, resulta razonable pensar que la estructura más adecuada para el abordaje en Salud Mental es ecológica (la persona y su entorno) basada en una atención articulada e intersectorial que se canaliza desde la atención primaria, partiendo de un nivel no especializado - con la adecuada orientación del Terapeuta Ocupacional en cuanto estilos de vida -, funcionalmente articulada con la atención y el control de riesgos - en este caso derivados de la ocupación - lo cual garantiza una continuidad en la atención y una ampliación considerable de cobertura concediendo a la educación de la comunidad una gran preponderancia y cuyos resultados en términos de responsabilidad y cuidado de su calidad de vida se verán en un mediano plazo.

La propuesta no significa ni desaparecer el hospital psiquiátrico ni considerar los programas no especializados como un complemento del hospital sino una centración en aspectos psicosociales más amplios a través de una red que incluya agentes dentro y fuera del sector salud, con descentralización e integración de elementos terapéuticos que hagan progresivamente innecesario el hospital psiquiátrico.

En esta perspectiva los programas de Salud Mental involucran diversos sectores como son la salud, el trabajo, la vivienda; la seguridad y el bienestar social, enfocados a solucionar los problemas prioritarios y teniendo en cuenta las consecuencias que para la Salud Mental tienen estas políticas. Para el Terapeuta Ocupacional resulta entonces imposible proyectar acciones donde el entorno, ecológicamente considerado, no juegue un papel preponderante.

En este orden de ideas es indispensable hacer esfuerzos para mejorar la calidad de la formación en Salud Mental de los Terapeutas Ocupacionales, pues a ellos corresponde la preparación del recurso comunitario en un aspecto fundamental como son sus rutinas ocupacionales. Las mejoras en la enseñanza deben contener también la aplicación de nuevos enfoques en rehabilitación comunitaria incluyendo intervenciones en la familia, en el sitio de trabajo y en las redes de apoyo social, además de incorporar los avances a nivel internacional, las experiencias locales y comunitarias y el mejoramiento de los estándares de atención.

\section{Algunas Sugerencias Programáticas de Intervención}

Los programas de promoción y prevención deben involucrar al equipo 
rehabilitador con problemas propios de nuestra realidad social como son la violencia, el estrés en las ciudades, los problemas de la población joven, la inactividad de los ancianos, el deterioro de los valores, la discriminación de la mujer y la violencia doméstica entre otros; mediante investigaciones y caracterizaciones a nivel comunitario que faciliten priorizar los problemas y evaluar el impacto de las acciones.

Este trabajo permite también establecer redes de apoyo social, impartir educación sobre aspectos específicos y lograr soluciones a determinados problemas.

Además de la necesaria intervención de los terapeutas en los programas de Salud Mental, se hace cada vez más necesario que participen en los programas contra la violencia a todos los niveles, en los de las víctimas de violencia colectiva como campos de desplazados, hogares sustitutos, programas de bienestar, lugares de reclusión, indigentes y otros.

De igual modo es necesario crear programas comunitarios para conformar unas rutinas cotidianas satisfactorias y productivas y en casos específicos establecer programas productivos para personas incapaces de lograr un empleo competitivo, obviamente con el apoyo psiquíatrico y psicológico que requieran pero abandonando el estereotipo que fuera de la hospitalización completa y el Hospital Día no habría más alternativas.

Una atención especial debe darse al abuso de sustancias como el alcohol y las drogas para desarrollar programas específicos que incluyan la información, la educación, la intervención acorde con los patrones de consumo, distribución y culturales acompañados de un seguimiento estricto a los resultados obtenidos.

Todo esto supone un amplio panorama investigativo y de aplicación para el terapeuta que encuentra que su responsabilidad se hace mayor en la medida en que la sociedad es más compleja y hemos avanzado considerablemente en la preservación fisica de la vida. 


\section{Bibliografía}

Nuevos Sistemas de Atención en Salud Mental: Evaluación e Investigación. Compiladores: García González José, Aparicio Basauri Victor. Consejería de Seguridad y Servicios Sociales. Asturias 1.990

Stein, Leonar MD, Test, Mary Ann Phd. Alternative to Mental Hospital Treatment. Archives of General Psichiatry. Vol 37. April 1980.

Comité Consultịo Nacional de Discapacidad y Derecho. Vicepresidencia de la República. 1.996.

OPS. Restrepo, Helena de las políticas de promoción de la salud en la OPS. Bogotá, Octubre 1.992. 